\title{
A Transient Directing Group Strategy Enables Enantioselective Multicomponent Organofluorine Synthesis
}

\author{
Authors: Zhonglin Liu†, Lucas J. Oxtoby†, Mingyu Liu, Zi-Qi Li, Van T. Tran, Yang Gao, \\ Keary M. Engle*.
}

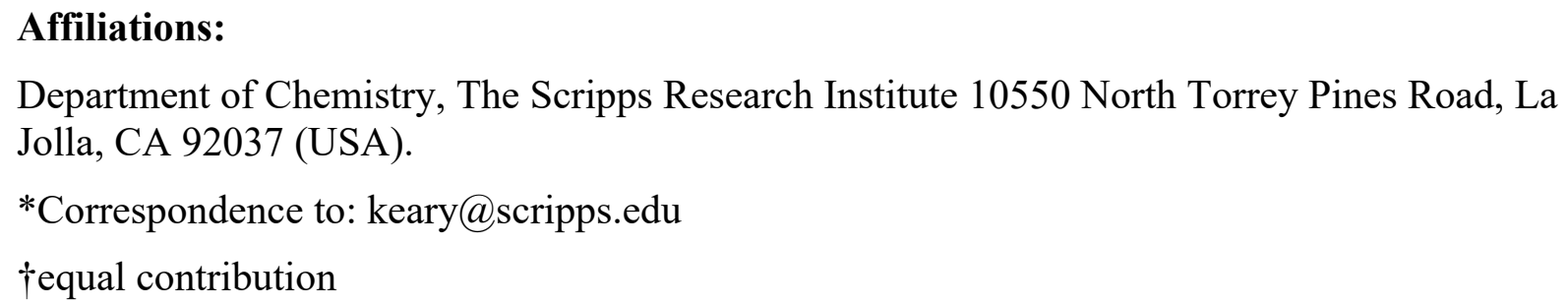

Abstract: The vicinal fluorofunctionalization of alkenes represents an expedient strategy for converting feedstock olefins into valuable fluorinated molecules and as such has garnered significant attention from the synthetic community; however, current methods remain limited in terms of scope and selectivity. Here we report the site-selective palladium-catalyzed threecomponent coupling of alkenylbenzaldehydes, arylboronic acids, and $N$-fluoro-2,4,6trimethylpyridinium hexafluorophosphate facilitated by a transient directing group. The synthetically enabling methodology constructs vicinal stereocenters with excellent regio-, diastereo-, and enantioselectivities, forging products that map onto bioactive compounds.

Main Text: The incorporation of carbon-fluorine $(\mathrm{C}-\mathrm{F})$ bonds into drug molecules can often improve their pharmacokinetic properties, including increasing oral bioavailability, protein 20 binding affinities, and metabolic stability, especially in the case of replacement of benzylic C-H bonds prone to metabolic oxidation ${ }^{1,2}$. As such, the development of strategies that enable the enantioselective formation of $\mathrm{C}-\mathrm{F}$ bonds has become a major research area of both industrial and academic importance in recent years ${ }^{3-5}$. In particular, intermolecular 1,2-carbofluorination of alkenes is an attractive transformation as it allows for the conversion of alkene feedstocks into fluorinated molecules with potential applications in the pharmaceutical, agrochemical, and material sectors ${ }^{6}$; however, this type of transformation remains challenging to execute due to issues with regio-, stereo-, and chemoselectivity. In early work, the groups of $\mathrm{Ma}^{7}$, Gagné ${ }^{8}$, Alexakis ${ }^{9}$, and Gouverneur ${ }^{10}$ reported pioneering examples of asymmetric fluorocyclizations of prochiral alkenes, in which a functional group tethered to the alkene reacts in the cyclization process.

More recently, Toste and coworkers have reported an elegant series of intermolecular (threecomponent) asymmetric arylfluorination reactions to construct chiral benzyl fluorides using palladium/N,N-ligand systems. This strategy has been used for both 1,1-arylfluorination (where regioselectivity is governed by substrate electronics) ${ }^{11,12}$ and 1,2-arylfluorination (where selectivity is governed by substrate directivity $)^{13}$. While the aforementioned work represents a great deal of progress, significant limitations remain. Palladium-catalyzed arylfluorination reactions are sensitive to alkene substitution patterns; for instance, disubstituted alkenes require double activation to enhance reactivity ${ }^{14}$, and no existing methods are able to construct fully substituted $\mathrm{C}\left(s p^{3}\right)-\mathrm{F}$ or $\mathrm{C}\left(s p^{3}\right)-\mathrm{Ar}$ stereocenters. Additionally, achieving high levels of pathway selectivity for a given substrate class (favoring 1,2-arylfluorination over 1,1-arylfluorination, $\beta$ hydride elimination, or other side reactions) often requires extensive ligand optimization and the use of potentially synthetically restrictive directing groups ${ }^{15}$. 
With the previous efforts in mind, we wondered if we could address these issues by implementing a chiral transient directing group (TDG) strategy. The viability of catalytic TDGs has previously been established in several mechanistically distinct transition-metal catalyzed reactions, including notably in the field of $\mathrm{C}-\mathrm{H}$ activation ${ }^{16}$; however, the scope of transiently directed asymmetric alkene functionalizations remains quite limited, and 1,2-difunctionalization reactions using a TDG approach remain unknown. Herein, we report a highly enantioselective 1,2-arylfluorination of alkenyl benzaldehydes that is able to form two vicinal chiral centers, including fully substituted $\mathrm{C}\left(s p^{3}\right)-\mathrm{F}$ and $\mathrm{C}\left(s p^{3}\right)-\mathrm{Ar}$ stereocenters, in synthetically useful yields with broad functional group tolerance.

To reduce this idea to practice, we based our initial reaction design on our recently reported enantioselective reductive Heck hydroarylation of alkenyl benzaldehydes using an amino acid $\mathrm{TDG}^{17}$. In our previous work, a stabilized alkylpalladium(II) intermediate is intercepted with formate, which decarboxylates to generate an alkylpalladium(II)-hydride species that subsequently undergoes reductive elimination. In the case of the envisioned transiently directed arylfluorination, the stabilized alkylpalladium(II) intermediate would react with a fluorinating oxidant (an $\left[\mathrm{F}^{+}\right]$reagent) to generate a palladium(IV) species that could undergo $\mathrm{C}-\mathrm{F}$ reductive elimination ${ }^{18}$. This seemingly simple extension is fraught with challenges, including undesired oxidation of the native aldehyde functional handle by $\left[\mathrm{F}^{+}\right]$, competitive homocoupling of arylboronic acids in the presence of palladium(II) and oxidant, and deleterious interactions between the TDG and $\left[\mathrm{F}^{+}\right]$.

In a series of pilot experiments, we found that L-tert-leucine (the optimal TDG in our previous system) did not lead to product formation, suggesting that a different TDG design was needed to support palladium(IV) formation. In their study on enantioselective $\mathrm{C}-\mathrm{H}$ fluorination of electrondeficient benzaldehydes, $\mathrm{Yu}$ and coworkers found that switching from an LX-type amino acid to an $\mathrm{L}_{2}$-type $\alpha$-amino amide TDG promoted $\mathrm{C}-\mathrm{F}$ reductive elimination through formation of a pentacoordinate cationic $\operatorname{Pd}(\mathrm{IV})$ complex ${ }^{19}$. We reasoned our system would benefit from the same effect and carried out a new screen using a library of TDGs that offered the potential of $\mathrm{L}_{2}$-type binding after aldehyde condensation. An initial hit was observed reacting alkene starting material 1 with $N$-fluoro-2,4,6-trimethylpyridinium salt $\left(\left[\mathrm{F}^{+}\right]\right)$and phenylboronic acid in the presence of a palladium(II) catalyst, previously unreported TDG-A, silver fluoride additive, and water in a 2:1 mixture of $\mathrm{DCM} / \mathrm{MeCN}$. A variety of unproductive side reactions were observed, including formation of palladium black and decomposition of the $\left[\mathrm{F}^{+}\right]$reagent and benzaldehyde SM (see SI for details). Because it was expected that most of the components would have strong interactions with each other that would impact the final yield of the reaction, our system appeared better suited for optimization via Design of Experiments (DoE) as opposed to typical "One Variable At a Time" (OVAT) screening. Despite its advantages over OVAT and its widespread adoption in industry, DoE remains underutilized in academic research ${ }^{20,21}$.

We elected to use a modified Definitive Screening Design (DSD) (Table 1), which allowed us to develop a linear regression model that describes the sensitivity of a response (in this case reaction yield) to a variety of input parameters with continuous levels (reagent loadings) ${ }^{22}$. The high- and low-end values for each reagent loading in the subsequent experiments were set based on what we expected to be the most extreme values the reaction would tolerate. We then ran just 18 experiments using values within those ranges to train a model, which subsequently predicted conditions that more than doubled our initial yield. While the arylboronic acid loading of 4.67 equivalents is relatively high, we deemed this acceptable in our system, as the arylboronic acid 
was not among the most valuable components of the reaction. It should be noted that while this technique can be used for rapid optimization and identification of major interactions, it does not suggest an explanation for why a given response is dependent on a particular input parameter; moreover, the DSD model does not necessarily accurately predict reaction performance in totally different chemical space. Multiple powerful techniques have emerged for the statistical prediction of out-of-sample reactions, including multivariate regression of physical organic descriptors (23) and machine-learning based chemical featurization approaches ${ }^{24,25}$, but these methods rely on much larger data sets with more input parameters. Our model translated well to other disubstituted and trisubstituted alkenes, but we did find that it was necessary to change TDGs for terminal alkene 10 substrates (see SI for details).

Having optimized the conditions, we began investigating the scope of alkene substitution. The reaction with 1,2,2-trisubstituted alkenes to form quaternary carbon-aryl bonds proceeded in moderate to good yields with excellent enantioselectivity and broad functional group tolerance. A number of approaches for the enantioselective formation of fully substituted $\mathrm{C}\left(s p^{3}\right)-\mathrm{F}$ stereocenters have been developed ${ }^{26}$, including enolate-type reactions of carbonyl derivatives with electrophilic fluorinating reagents ${ }^{27-34}$, enolate-type reactions of $\alpha$-fluorinated carbonyl derivatives with arylating/alkylating reagents ${ }^{35-39}$, the reaction of alkenes with electrophilic fluorinating reagents to form allylic fluoride products ${ }^{40-45}$, iridium-catalyzed allylic substitution reactions of alkenyl fluorides ${ }^{46-48}$, and the redox-relay palladium-catalyzed enantioselective Heck reaction of acyclic alkenyl fluorides with arylboronic acids ${ }^{49}$. Successful reaction of 1,1,2-trisubstituted alkene substrates with our conditions would form tertiary $\mathrm{C}\left(s p^{3}\right)$-F stereocenters, which would be a valuable addition to previously reported methods; however, this requires an unprecedented asymmetric formation of a fully substituted $\mathrm{C}\left(s p^{3}\right)-\mathrm{F}$ stereocenter through $\mathrm{C}-\mathrm{F}$ reductive elimination. Gratifyingly, the reaction with 1,1,2-trisubstituted alkenes was able to form tertiary $\mathrm{C}\left(s p^{3}\right)$-F stereocenters, with somewhat lower, but still high enantioselectivity ranging from 90 96\% ee. Next, we explored the effects of other alkene and benzaldehyde substitutions on the reaction. Both electron-rich and electron-poor benzaldehydes gave good yields with excellent enantioselectivity, with performance being similar between $E$ - and $Z$-isomers of the alkene starting material ( $2 \mathbf{y}$ to $\mathbf{3 y}$ and $\mathbf{2 z}$ to $\mathbf{3 z}$, respectively). Unfortunately, the method is ineffective for alkenes that are tetrasubstituted or whose functionalization results in dearomatization (see SI for all examples tested).

We explored the scope of arylboronic acids with 1a as the model alkene substrate. Both electronrich and electron-poor para-substituted boronic acids afforded the desired products in good yields with excellent enantioselectivity. Arylboronic acids with electron-withdrawing meta-substituents (4f) performed markedly worse than those with electron-donating substituents (4g), requiring either prolonged reaction time or higher temperatures. The reaction did proceed with benzofuran (3h) and benzodioxole (4i) boronic acids, but did not tolerate other heterocycles, including substituted pyridines and pyrazoles. Additional limitations include both alkenyl and alkyl boronic acids. In order to demonstrate the potential synthetic utility of the reaction, multiple 40 diversifications were performed. Both oxidation (5a) and reduction (5c) of the aldehyde proceeded in high yields while maintaining the high ee of $\mathbf{4 a}$. Olefination $(\mathbf{5 b})$ and decarbonylation $(\mathbf{5 d})$ gave the desired products in moderate yields with little to no erosion in ee. To further demonstrate the synthetically enabling nature of this method, we targeted the synthesis of the bioactive compound MCJ001F $\mathrm{F}^{50,51}$. The reported patent route to the $(R, R)$-stereoisomer involves a chiral resolution of 45 a racemic intermediate and late-stage separation of diastereomers in a 10-step sequence. In contrast, our procedure is both enantio- and diastereoselective, affording MCJ001F-RR in fewer 
steps from commercially available materials and in overall higher yield. By starting from the opposite alkene isomer (Z-configured), we were also able to prepare the $(R, S)$-stereoisomer in higher overall yield than the reported patent route (see SI for a full comparison to previous syntheses).

In an attempt to gain insight into the reaction mechanism, we performed several control reactions and kinetic experiments. First, we confirmed that the TDG was essential by excluding it from a standard reaction with $\mathbf{7 f}$ as well as subjecting ester $\mathbf{7 e}$, which cannot condense with the TDG, to standard conditions. In both cases, only recovered starting material was observed. Next, we confirmed the reaction does not occur through $\mathrm{C}-\mathrm{H}$ activation by subjecting substrate $\mathbf{7 g}$ to our standard conditions and observing that it was unreactive. The role of AgF was also briefly considered. While the reaction of model substrate 1a does proceed without silver fluoride, the reaction only reaches roughly $40 \%$ yield by ${ }^{1} \mathrm{H}$ NMR, with the remaining mass balance of $1 \mathrm{a}$ being decomposed material. We observe the formation of palladium black when stirring a mixture of 1a, various loadings of TDG-A, and $\mathrm{Pd}(\mathrm{MeCN})_{4}\left(\mathrm{BF}_{4}\right)_{2}(10 \mathrm{~mol} \%)$ in the standard solvent conditions, raising the possibility of catalyst deactivation in the reaction system. Palladium black was qualitatively observed forming over the course of roughly 6 hours at $35^{\circ} \mathrm{C}$ for loadings of TDG higher than $75 \mathrm{~mol} \%$. Competitive homocoupling of the boronic acid (confirmed by GC-MS) helps explain the high amount of boronic acid required and the potential beneficial effect of $\operatorname{Ag}(\mathrm{I})$ for reoxidation of inactive $\operatorname{Pd}(0)$.

Experiments aimed at establishing the robustness of the palladium catalyst and the concentration dependencies of the reaction components were carried out according to the "same-excess" and "different-excess" protocols of Reaction Progress Kinetic Analysis (RPKA), respectively ${ }^{52}$. RPKA allows for visual analysis of the kinetic data over the entire time course of the reaction at synthetically relevant conditions. To identify if there is significant catalyst deactivation or product inhibition in our system, we compared temporal concentration profiles ([1a] vs. time) of reactions carried out under same-excess conditions (Figure 2). This comparison is facilitated by shifting the profile of a reaction started at lower concentration to the right on the timescale until the first point overlays with the second profile (the standard conditions $)^{53}$. For any point on the time-adjusted profile, both reactions will have the same concentration of starting materials, but different concentration of product and a different number of turnovers completed by the catalyst. The lack of overlay between these profiles indicates that product inhibition or catalyst deactivation is occurring. These possibilities can be discerned by running a third experiment with the amount of product generated by the reaction until this reaction point added. This reaction is identical by composition, but the catalyst has completed fewer turnovers; therefore, the lack of overlay in this case is indicative of mild catalyst deactivation.

Having confirmed the presence of catalyst deactivation, initial rates from reaction progress profiles were employed to probe concentration dependencies of the reaction components. A series of "different-excess" experiments, in which the order in a given substrate is determined by comparison of reactions with different concentrations of that specific substrate but identical concentrations of other reaction components showed the reaction to have a positive dependence on [Pd] and a negative rate dependence on [TDG]. Studies established the absence of a nonlinear effect of TDG ee, showing that product ee varies linearly with TDG ee. This, coupled with an observed first-order dependence on palladium, confirms the absence of Pd dimers or other higher order Pd species either on or off the cycle, but does not preclude the possibility of monomeric offcycle species $^{54}$. The reaction was shown to be zero-order in water, silver fluoride, and alkene. The 
initial rates for reactions with lower $\left[\mathrm{PhB}(\mathrm{OH})_{2}\right]$ or lower $\left[\mathrm{F}^{+}\right]$were reduced compared to the reaction run under standard conditions, suggesting a positive rate dependence; however, when comparing increased concentrations of these reagent during the "different excess" experiments, the resulting rates showed an apparent zero-order dependence in $\left[\mathrm{F}^{+}\right]$and boronic acid, pointing to saturation kinetics in both of these components.

Taken together, the kinetics data are consistent with the mechanism proposed in Figure 2 where transmetalation is turnover-limiting at low concentrations of boronic acid and oxidative addition is turnover-limiting at low concentrations of $\left[\mathrm{F}^{+}\right]$. A zero-order dependence in all components under standard conditions is consistent with the $\mathrm{C}-\mathrm{F}$ reductive elimination step being turnoverlimiting. Negative-order rate dependence on the concentration of TDG is consistent with the TDG mediating decomposition of $\left[\mathrm{F}^{+}\right]$or potentially forming an off-cycle $\mathrm{TDG} \cdot \mathrm{Pd}^{\mathrm{II}}$ complex (the nonlinear experiments suggest this off-cycle intermediate would be monometallic $)^{54}$. Analogous to our previous studies, we hypothesize that the chiral TDG mediates enantiodetermining migratory insertion by attenuating geometric distortion in the favored metallatricyclic transition state $^{17}$. The kinetics data as a whole underscore the mechanistic complexity of this TDG-mediated arylfluorination reaction and highlights the value of DoE for optimization of complicated dual catalytic systems of this type in the absence of a detailed a priori mechanistic picture. Not only does the highly enantioselective 1,2-arylfluorination of alkenyl benzaldehydes presented here allow expedient access to organofluorine compounds that are otherwise difficult to prepare; its successful development sets the stage for expansion of chiral TDG strategies across increasingly diverse alkene difunctionalization reactions.

\section{References:}

1. Purser, S., Moore, P. R., Swallow, S. \& Gouverneur, V. Fluorine in medicinal chemistry. Chem. Soc. Rev. 37, 320-330 (2008).

2. Gillis, E. P., Eastman, K. J., Hill, M. D., Donnelly, D. J. \& Meanwell, N. A. Applications of Fluorine in Medicinal Chemistry. J. Med. Chem. 58, 8315-8359 (2015).

3. Ma, J. A. \& Cahard, D. Asymmetric fluorination, trifluoromethylation, and perfluoroalkylation reactions. Chem. Rev. 104, 6119-6146 (2004).

4. Brunet, V. A. \& O'Hagan, D. Catalytic asymmetric fluorination comes of age. Angew. Chem. In. Ed. 47, 1179-1182 (2008).

5. Yang, X., Wu, T., Phipps, R. J. \& Toste, F. D. Advances in catalytic enantioselective fluorination, mono-, di-, and trifluoromethylation, and trifluoromethylthiolation reactions. Chem. Rev. 115, 826-870 (2015).

6. Wolstenhulme, J. R. \& Gouverneur, V. Asymmetric fluorocyclizations of alkenes. Acc. Chem. Res. 47, 3560-3570 (2014).

7. Nie, J., Zhu, H. W., Cui, H. F., Hua, M. Q. \& Ma, J. A. Catalytic stereoselective synthesis of highly substituted indanones via tandem Nazarov cyclization and electrophilic fluorination trapping. Org. Lett. 9, 3053-3056 (2007).

40 8. Cochrane, N. A., Nguyen, H. \& Gagne, M. R. Catalytic enantioselective cyclization and C3fluorination of polyenes. J. Am. Chem. Soc. 135, 628-63 (2013).

9. Romanov-Michailidis, F., Guenee, L. \& Alexakis, A. Enantioselective organocatalytic fluorination-induced Wagner-Meerwein rearrangement. Angew. Chem. In. Ed. 52, 9266-9270 (2013).

10. Wolstenhulme, J. R. et al. Asymmetric electrophilic fluorocyclization with carbon nucleophiles. 
Angew. Chem. In. Ed. 52, 9796-9800 (2013).

11. He, Y., Yang, Z., Thornbury, R. T. \& Toste, F. D. Palladium-catalyzed enantioselective 1,1fluoroarylation of aminoalkenes. J. Am. Chem. Soc. 137, 12207-12210 (2015).

12. Miro, J., Del Pozo, C., Toste, F. D. \& Fustero, S. Enantioselective Palladium-Catalyzed Oxidative $\beta, \beta$-Fluoroarylation of $\alpha, \beta$-Unsaturated Carbonyl Derivatives. Angew. Chem. In. Ed. 55, 9045-9049 (2016).

13. Talbot, E. P., Fernandes Tde, A., McKenna, J. M. \& Toste, F. D. Asymmetric palladiumcatalyzed directed intermolecular fluoroarylation of styrenes. J. Am. Chem. Soc. 136, 41014104 (2014).

14. Xi, Y., Wang, C., Zhang, Q., Qu, J. \& Chen, Y. Palladium-Catalyzed Regio-, Diastereo-, and Enantioselective 1,2-Arylfluorination of Internal Enamides. Angew. Chem. In. Ed., 59 (2020).

15. Thornbury, R. T. et al. The development and mechanistic investigation of a palladiumcatalyzed 1,3-arylfluorination of chromenes. Chem. Sci. 8, 2890-2897 (2017).

16. Higham, J. I. \& Bull, J. A. Transient imine directing groups for the C-H functionalisation of aldehydes, ketones and amines: an update 2018-2020. Org. Biomol. Chem. 18, 7291-7315 (2020).

17. Oxtoby, L. J. et al. A transient-directing-group strategy enables enantioselective reductive Heck hydroarylation of alkenes. Angew. Chem. In. Ed. 59, 8885-8890 (2020).

18. Kalyani, D., Satterfield, A. D. \& Sanford, M. S. Palladium-catalyzed oxidative arylhalogenation of alkenes: synthetic scope and mechanistic insights. J. Am. Chem. Soc. 132, 8419-8427 (2010).

19. Park, H., Verma, P., Hong, K. \& Yu, J. Q. Controlling Pd ${ }^{\mathrm{IV}}$ reductive elimination pathways enables $\mathrm{Pd}^{\mathrm{II}}$-catalysed enantioselective $\mathrm{C}\left(\mathrm{sp}^{3}\right)$-H fluorination. Nat. Chem. 10, 755-762 (2018).

20. Leardi, R. Experimental design in chemistry: A tutorial. Anal. Chim. Acta. 652, 161-172 (2009).

21. Lendrem, D., Owen, M. \& Godbert, S. DOE (Design of Experiments) in Development Chemistry: Potential Obstacles. Org. Process Res. Dev. 5, 324-327 (2001).

22. Jones, B. \& Nachtsheim, C. J. A Class of Three-Level Designs for Definitive Screening in the Presence of Second-Order Effects. J. Qual. Technol. 43, 1-15 (2011).

23. Reid, J. P. \& Sigman, M. S. Holistic prediction of enantioselectivity in asymmetric catalysis. Nature 571, 343-348 (2019).

24. Ahneman, D. T., Estrada, J. G., Lin, S., Dreher, S. D. \& Doyle, A. G. Predicting reaction performance in C-N cross-coupling using machine learning. Science 360, 186-190 (2018).

25. Zahrt, A. F. et al. Prediction of higher-selectivity catalysts by computer-driven workflow and machine learning. Science 363 (2019).

35 26. Zhu, Y. et al. Modern Approaches for Asymmetric Construction of Carbon-Fluorine Quaternary Stereogenic Centers: Synthetic Challenges and Pharmaceutical Needs. Chem. Rev. 118, 3887-3964 (2018).

27. Shibata, N., Suzuki, E., Asahi, T. \& Shiro, M. Enantioselective fluorination mediated by cinchona alkaloid derivatives/Selectfluor combinations: reaction scope and structural information for $N$-fluorocinchona alkaloids. J. Am. Chem. Soc. 123, $7001-7009$ (2001).

28. Mohar, B., Baudoux, J., Plaquevent, J.-C. \& Cahard, D. Electrophilic Fluorination Mediated by Cinchona Alkaloids: Highly Enantioselective Synthesis of $\alpha$-Fluoro- $\alpha$-phenylglycine Derivatives. Angew. Chem. In. Ed. 40, 4214-4216 (2001).

29. Marigo, M., Fielenbach, D., Braunton, A., Kjaersgaard, A. \& Jorgensen, K. A. Enantioselective formation of stereogenic carbon-fluorine centers by a simple catalytic method. Angew. Chem. In. Ed. 44, 3703-3706 (2005). 
30. Steiner, D. D., Mase, N. \& Barbas, C. F., 3rd. Direct asymmetric $\alpha$-fluorination of aldehydes. Angew. Chem. In. Ed. 44, 3706-3710 (2005).

31. Shibatomi, K., Kitahara, K., Okimi, T., Abe, Y. \& Iwasa, S. Enantioselective fluorination of $\alpha-$ branched aldehydes and subsequent conversion to $\alpha$-hydroxyacetals via stereospecific C-F bond cleavage. Chem. Sci. 7, 1388-1392 (2016).

32. You, Y., Zhang, L. \& Luo, S. Reagent-controlled enantioselectivity switch for the asymmetric fluorination of $\beta$-ketocarbonyls by chiral primary amine catalysis. Chem. Sci. 8, 621-626 (2017).

33. Shibata, N. et al. Highly enantioselective catalytic fluorination and chlorination reactions of carbonyl compounds capable of two-point binding. Angew. Chem. In. Ed. 44, 4204-4207 (2005).

34. Reddy, D. S. et al. Desymmetrization-like catalytic enantioselective fluorination of malonates and its application to pharmaceutically attractive molecules. Angew. Chem. In. Ed. 47, 164168 (2008).

35. Jiao, Z. et al. Palladium-catalyzed enantioselective $\alpha$-arylation of $\alpha$-fluoroketones. J. Am. Chem. Soc. 138, 15980-15986 (2016).

36. Belanger, E., Cantin, K., Messe, O., Tremblay, M. \& Paquin, J. F. Enantioselective Pdcatalyzed allylation reaction of fluorinated silyl enol ethers. J. Am. Chem. Soc. 129, 1034-1035 (2007).

37. Liang, Y. \& Fu, G. C. Catalytic asymmetric synthesis of tertiary alkyl fluorides: Negishi crosscouplings of racemic $\alpha, \alpha$-dihaloketones. J. Am. Chem. Soc. 136, 5520-5524 (2014).

38. Han, X., Kwiatkowski, J., Xue, F., Huang, K. W. \& Lu, Y. Asymmetric Mannich reaction of fluorinated ketoesters with a tryptophan-derived bifunctional thiourea catalyst. Angew. Chem. In. Ed. 48, 7604-7607 (2009).

39. Xie, C., Wu, L., Han, J., Soloshonok, V. A. \& Pan, Y. Assembly of Fluorinated Quaternary Stereogenic Centers through Catalytic Enantioselective Detrifluoroacetylative Aldol Reactions. Angew. Chem. In. Ed. 54, 6019-6023 (2015).

40. Ishimaru, T. et al. Cinchona alkaloid catalyzed enantioselective fluorination of allyl silanes, silyl enol ethers, and oxindoles. Angew. Chem. In. Ed. 47, 4157-4161 (2008).

41. $\mathrm{Wu}, \mathrm{J}$. et al. A combination of directing groups and chiral anion phase-transfer catalysis for enantioselective fluorination of alkenes. Proc. Natl Scad. Sci. 110, 13729-13733 (2013).

42. Lozano, O. et al. Organocatalyzed enantioselective fluorocyclizations. Angew. Chem. In. Ed. 50, 8105-8109 (2011).

43. Rauniyar, V., Lackner, A. D., Hamilton, G. L. \& Toste, F. D. Asymmetric electrophilic fluorination using an anionic chiral phase-transfer catalyst. Science 334, 1681-1684 (2011).

44. Shunatona, H. P., Fruh, N., Wang, Y. M., Rauniyar, V. \& Toste, F. D. Enantioselective fluoroamination: 1,4-addition to conjugated dienes using anionic phase-transfer catalysis. Angew. Chem. In. Ed. 52, 7724-7727 (2013).

45. Egami, H. et al. Dianionic Phase-Transfer Catalyst for Asymmetric Fluoro-cyclization. J. Am. Chem. Soc. 140, 2785-2788 (2018).

46. Butcher, T. W. \& Hartwig, J. F. Enantioselective Synthesis of Tertiary Allylic Fluorides by Iridium-Catalyzed Allylic Fluoroalkylation. Angew. Chem. In. Ed. 57, 13125-13129 (2018).

47. He, Z. T., Jiang, X. \& Hartwig, J. F. Stereodivergent Construction of Tertiary Fluorides in Vicinal Stereogenic Pairs by Allylic Substitution with Iridium and Copper Catalysts. J. Am. Chem. Soc. 141, 13066-13073 (2019).

48. Butcher, T. W. et al. Desymmetrization of difluoromethylene groups by C-F bond activation. 
Nature 583, 548-553 (2020).

49. Liu, J., Yuan, Q., Toste, F. D. \& Sigman, M. S. Enantioselective construction of remote tertiary carbon-fluorine bonds. Nat. Chem. 11, 710-715 (2019).

50. Valenta, P., Carroll, P. J. \& Walsh, P. J. Stereoselective synthesis of $\beta$-hydroxy enamines, aminocyclopropanes, and 1,3-amino alcohols via asymmetric catalysis. J. Am. Chem. Soc. 132, 14179-14190 (2010).

51. Richelson, E., Fauq, A. H. Inhibiting neurotransmitter reuptake (2016), US 20160024044

52. Blackmond, D. G. Reaction progress kinetic analysis: a powerful methodology for mechanistic studies of complex catalytic reactions. Angew. Chem. In. Ed. 44, 4302-4320 (2005).

53. Baxter, R. D., Sale, D., Engle, K. M., Yu, J. Q. \& Blackmond, D. G. Mechanistic rationalization of unusual kinetics in Pd-catalyzed C-H olefination. J. Am. Chem. Soc. 134, 4600-4606 (2012).

54. Hill, D. E. et al. A General Protocol for Addressing Speciation of the Active Catalyst Applied to Ligand-Accelerated Enantioselective $\mathrm{C}\left(\mathrm{sp}^{3}\right)-\mathrm{H}$ Bond Arylation. ACS Catal. 8, 1528-1531 (2018).

Acknowledgments: We thank Dr. Dee-Hua Huang and Dr. Laura Pasternack for assistance with NMR spectroscopy. We also thank Dr. Gary J. Balaich (UCSD) for X-ray crystallographic analysis. Dr. Jason Chen, Brittany Sanchez, and Emily Sturgell (Scripps Research Automated Synthesis Facility) are acknowledged for HPLC, SFC, and HRMS analysis. We also thank Dr. Joseph Derosa and Dr. Donna Blackmond for helpful discussion. Funding: Financial support for this work was provided by the National Institutes of Health (R35GM125052) and the Cottrell Scholars program. Additional support was provided through an SIOC Fellowship (Z.L.) and a National Science Foundation Graduate Research Fellowship (NSF/DGE-184247, L.J.O.). Author contributions: Z.L., L.J.O., and K.M.E. conceived the concept. Z.L., L.J.O., M.L., Z.-Q.L., V.T.T., and Y.G. carried out the experiments. All of the authors analyzed the data. L.J.O. and K.M.E. wrote the manuscript with input from all authors. Competing interests: The authors declare no competing interests. Data and materials availability: All data is available in the main text or the supplementary materials. 


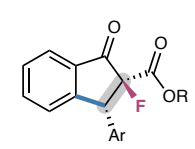

Ma (2007)

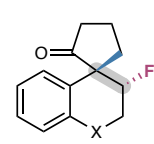

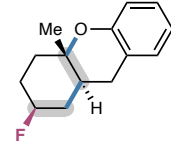

Gagné (2013)

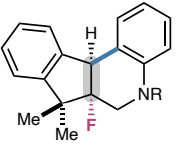

Gouverneur (2013)

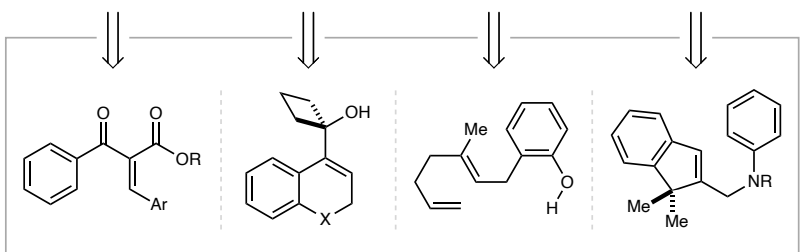

b previous approaches to Pd-catalyzed alkene arylfluorination
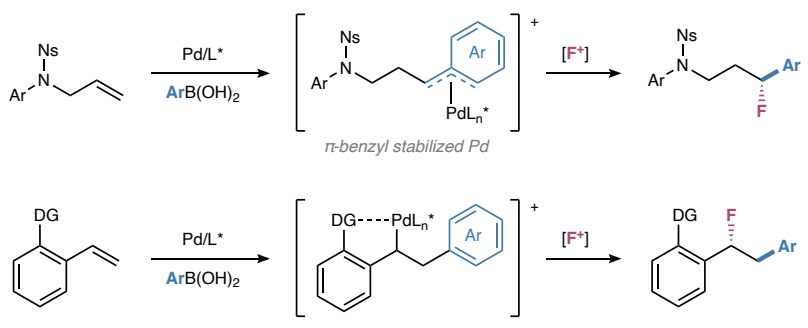

DG stabilized $P d$

C Pd-catalyzed alkene arylfluorination using TDG (this work)

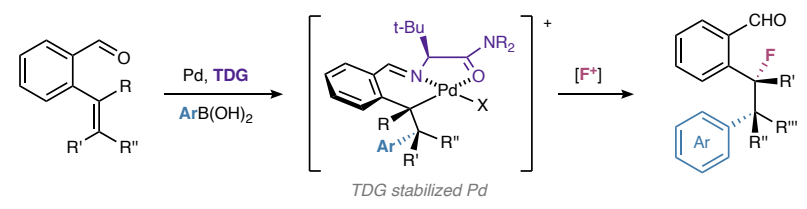

d optimization using a definitive screening design
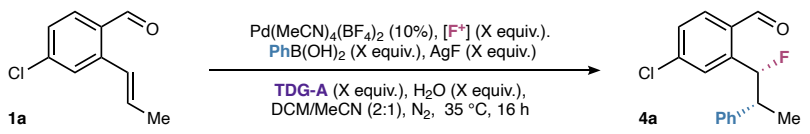

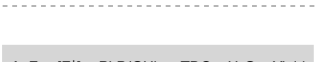

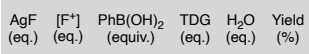

$\begin{array}{llllll}1.5 & 2.25 & 3.50 & 0.50 & 1.5 & 73.5 \\ 1.0 & 1.50 & 4.67 & 0.35 & 3.0 & 78.0\end{array}$

$\begin{array}{llllll}1.0 & 1.50 & 4.67 & 0.35 & 3.0 & 78.0 \\ 20 & 3.00 & 3.50 & 0.65 & 3.0 & 30.0\end{array}$

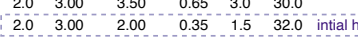

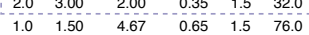

$\begin{array}{llllll}2.0 & 3.00 & 2.33 & 0.65 & 0.0 & 51.0\end{array}$

$\begin{array}{llllll}2.0 & 3.00 & 2.33 & 0.65 & 0.0 & 51.0 \\ 1.5 & 3.00 & 4.67 & 0.65 & 3.0 & 83.0\end{array}$

$\begin{array}{llllll}1.0 & 3.00 & 3.50 & 0.35 & 0.0 & 51.0\end{array}$

$\begin{array}{llllll}1.0 & 3.00 & 3.50 & 0.35 & 0.0 & 51.0 \\ 2.0 & 3.00 & 2.33 & 0.35 & 1.5 & 40.5\end{array}$

$\begin{array}{llllll}2.0 & 3.00 & 2.33 & 0.35 & 1.5 & 40.5 \\ 1.5 & 1.50 & 2.33 & 0.35 & 0.0 & 36.0\end{array}$

$\begin{array}{llllll}1.5 & 1.50 & 2.33 & 0.35 & 0.0 & 36.0 \\ 2.0 & 2.25 & 4.67 & 0.65 & 0.0 & 72.0\end{array}$

$\begin{array}{llllll}2.0 & 2.25 & 4.67 & 0.65 & 0.0 & 72.0 \\ 1.0 & 1.50 & 2.33 & 0.65 & 0.0 & 44.5\end{array}$

$\begin{array}{llllll}1.5 & 2.25 & 3.50 & 0.50 & 1.5 & 74.5\end{array}$

$\begin{array}{llllll}1.5 & 2.25 & 3.50 & 0.50 & 1.5 & 74.5 \\ 2.0 & 1.50 & 4.67 & 0.35 & 0.0 & 45.5\end{array}$

$\begin{array}{llllll}1.0 & 3.00 & 4.67 & 0.50 & 0.0 & 67.0\end{array}$

$\begin{array}{llllll}1.0 & 2.25 & 2.33 & 0.35 & 3.0 & 69.0\end{array}$

$\begin{array}{llllll}1.0 & 3.00 & 2.33 & 0.65 & 3.0 & 59.0\end{array}$

$\begin{array}{llllll}2.0 & 3.00 & 4.67 & 0.35 & 3.0 & 69.0\end{array}$

\begin{tabular}{llllll}
2.0 & 1.50 & 2.33 & 0.50 & 3.0 & 21.5 \\
\hline 1.0 & 2.25 & 4.67 & 0.50 & 3.0 & 90.0
\end{tabular}

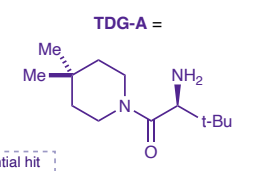

$\left[\mathrm{F}^{+}\right]=$

$\left[\mathrm{F}^{+}\right]=$

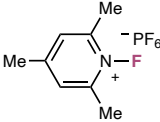

$\left[\mathrm{F}^{+}\right]$(equiv.) $\quad \mathrm{AgF}$ (equiv.)

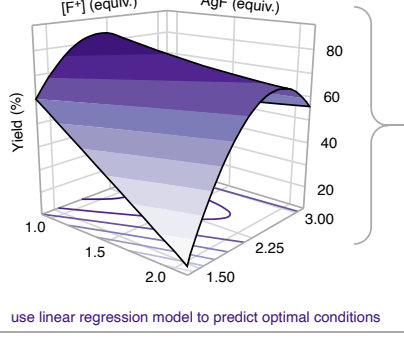

Figure 1 | Approaches to alkene fluorofunctionalization. a, Pioneering examples of asymmetric fluorocyclizations of alkenes. b, State of the art in palladium-catalyzed alkene arylfluorination. c, General depiction of an arylfluorination facilitated by an in situ formed imine using a chiral TDG strategy. d, Optimization of reaction conditions. 


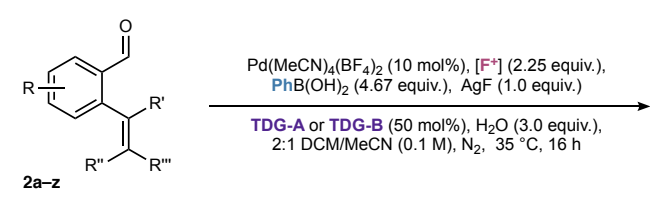

quaternary C-Ar formation
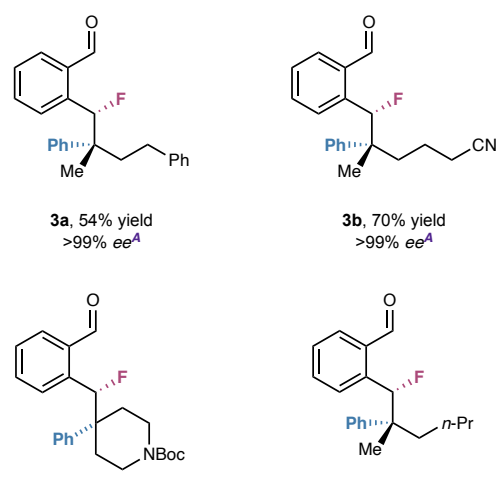

3a, $54 \%$ yield $3 \mathrm{e}, 56 \%$ yield
$>99 \% \mathrm{ee}^{A}$

\section{3b, $70 \%$ yield}

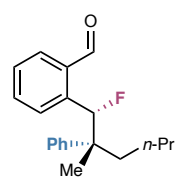

3f, $55 \%$ yield

$99 \% e e^{A}$

terminal alkenes

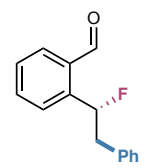

$3 \mathrm{~h}, 60 \%$ yield
$91 \% e^{B}$

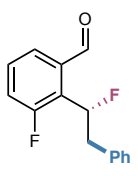

$\underset{3 i, 66 \% \text { yield }}{97 \% e^{B}}$

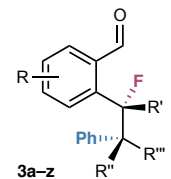

TDG-A = or

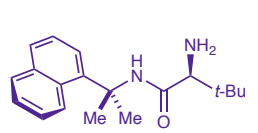

$\left[\mathrm{F}^{+}\right]=$

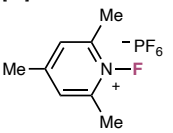

tertiary $C-F$ formation<smiles>CC(F)(Cc1ccccc1)c1ccccc1C=O</smiles>

3k, $33 \%$ yield $93 \% e e^{B}$

$3 c(R=E t) 50 \%,>99 \% e e^{A}$.<smiles>COc1ccc(COC(=O)C(C)C(C)c2ccccc2C=O)cc1</smiles>

3g, $65 \%$ yield

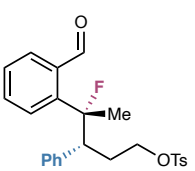

3n, $60 \%$ yield, $96 \% e e^{A}$

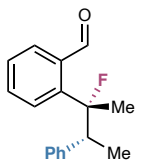

$3 \mathbf{p}$ (from E-isomer),

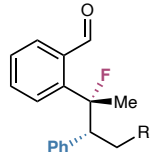

3 I (R = Cy) $75 \%, 95 \%$ ee $e^{A}$; $3 \mathbf{m}(\mathrm{R}=\mathrm{Bn}) 55 \%, 93 \% e e^{A}$

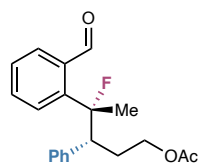

$30,66 \%$ yield $95 \% e e^{A}$

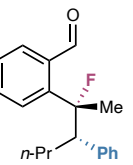

3q (from Z-isomer), $42 \%$ yield, $90 \%$ ee<smiles>COc1ccc([C+](F)[C@H](C)c2ccccc2)c(C=O)c1</smiles>
$3 \mathbf{r}, 83 \%$ yield
$98 \% e e^{A}$<smiles>COc1cc(C=O)c([C@H](F)C(c2ccccc2)c2ccccc2)cc1OC</smiles>

$3 \mathbf{v}, 85 \%$ yield
$96 \% e e^{A}$

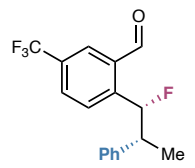

3s, $66 \%$ yield
$>99 \%$
a<smiles>CC(=O)c1ccc(C=O)c(C(F)[C@H](C)c2ccccc2)c1</smiles>

$3 \mathbf{w}, 80 \%$ yield
$>99 \% e e^{A}$

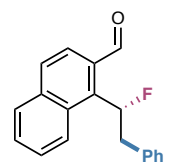

3j, $53 \%$ yield
$97 \% e e^{B}$ 1,2-disubstituted alkenes<smiles>Cc1cc([C+](F)[C@H](C)c2ccccc2)c(C=O)cc1F</smiles>

3 t, $52 \%$ yield
$>99 \% e e^{A}$<smiles>O=Cc1ccccc1[C@H](F)C(c1ccccc1)C1CC1</smiles>

$3 \mathbf{x}, 71 \%$ yield
$98 \% e e^{A}$

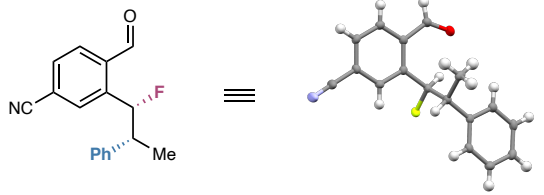

$3 \mathrm{u}, 41 \%$ yield
$>99 \% \mathrm{ee}^{A}$

$X$-ray crystal structure<smiles>O=Cc1ccccc1[C](F)[C](c1ccccc1)c1ccccc1</smiles><smiles>O=Cc1ccccc1[C](F)[C@H](F)c1ccccc1</smiles>

$3 y$ (from $E$-isomer),
$64 \%$ yield, $99 \% e e^{A}$
32 (from Z-isomer), $60 \%$ yield, $98 \% e e^{A}$

Table 1 | Alkenylbenzaldehyde scope of the arylfluorination reaction. Reactions were carried out on 0.1 mmol scale. All reported yields are isolated yields. The use of TDG-A or TDG-B is designated by a superscript A or B, respectively. 

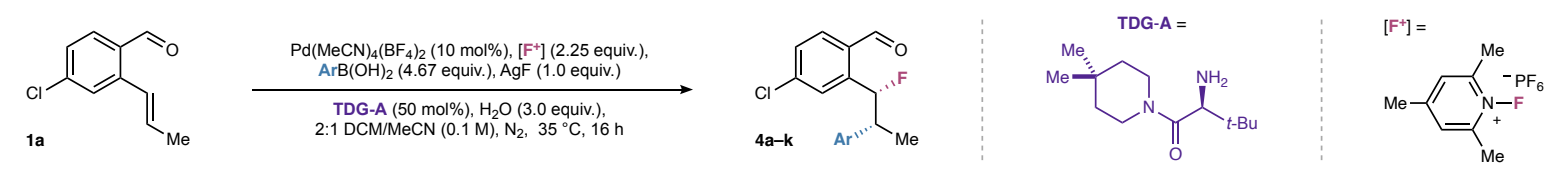

arylboronic acid scope<smiles>C[C@H](c1ccccc1)[C@@H](F)c1cc(Cl)ccc1C=O</smiles>

$4 a$
$90 \%$ yield, $>99 \%$ ee gram scale:
$84 \%$ yield, $99 \%$ ee<smiles>CCCc1ccc([C@@H](C)[C@H](F)c2cc(Cl)ccc2C=O)cc1</smiles>
$\underset{76 \%}{\mathbf{4 b}}$ yield, $>99 \%$ ee<smiles>COc1cccc([C@H](C)[C@H](F)c2cc(Cl)ccc2C=O)c1</smiles>

$\underset{63 \% \text { yield, } 98 \% \text { ee }}{\stackrel{4 g}{2}}$

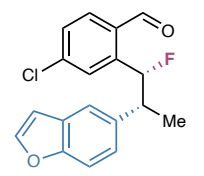

$\stackrel{4 \mathbf{h}}{50 \% \text { yield, }>99 \% \text { ee }}$

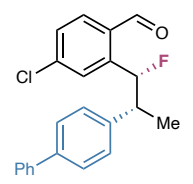

$\stackrel{4 c}{36 \%}$ yield, $98 \%$ ee

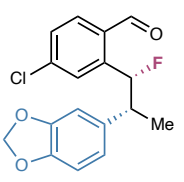

$\stackrel{4 i}{37 \%}$ yield, $94 \%$ ee

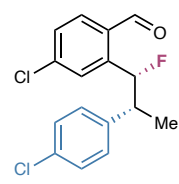

4d
$72 \%$ yield, $97 \%$ ee

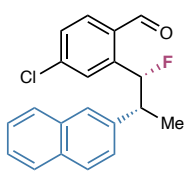

$\stackrel{4 j}{60 \% \text { yield, } 99 \% \text { ee }}$
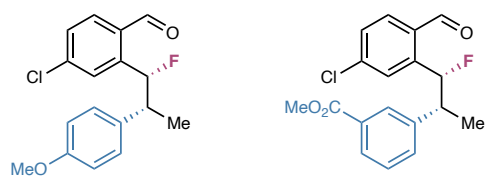

$4 \mathrm{e}$
$70 \%$ yield, $99 \%$ ee

$\stackrel{4 f}{34 \% \text { yield, } 94 \% \text { ee }}$

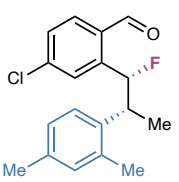

$\stackrel{4 k}{45 \% \text { yield, } 99 \% \text { ee }}$

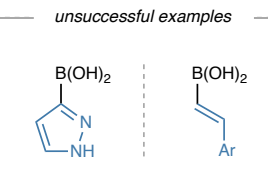

recovered starting material

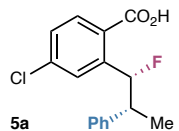

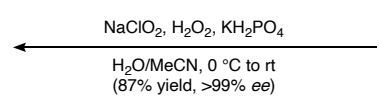

$\mathrm{H}_{2} \mathrm{O} / \mathrm{MeCN}, 0^{\circ} \mathrm{C}$ to rt
$(87 \%$ yield, $>99 \%$ ee $)$
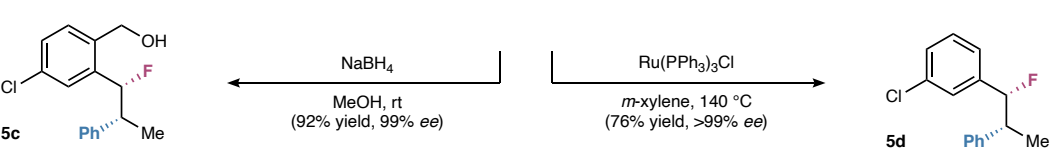

(Na)

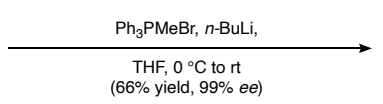

$\mathrm{THF}, 0{ }^{\circ} \mathrm{C}$ to $\mathrm{rt}$
(66\% yield, $99 \%$ ee)

synthesis of MCJ001F

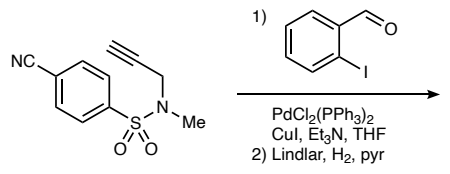

$6 \mathbf{a}$
(commercial)

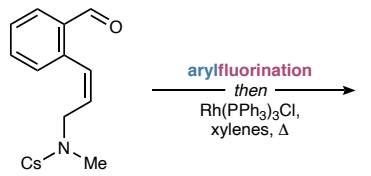

6b (83\% yield

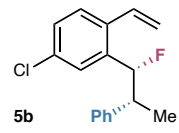

$5 b$

5

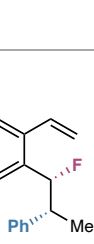



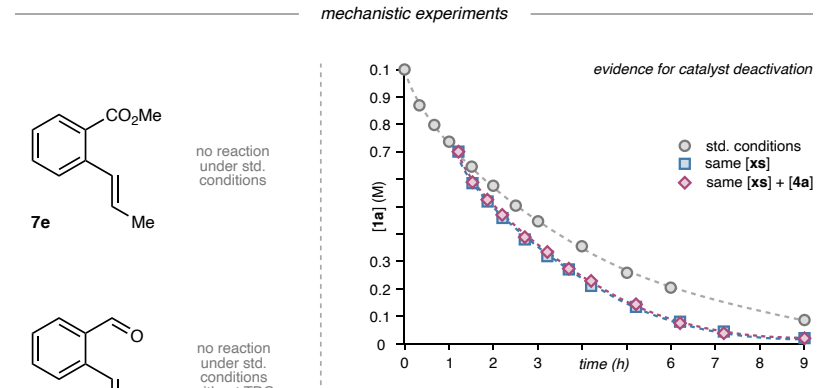

${ }_{7 f} \mathrm{~L}_{\mathrm{Me}}$
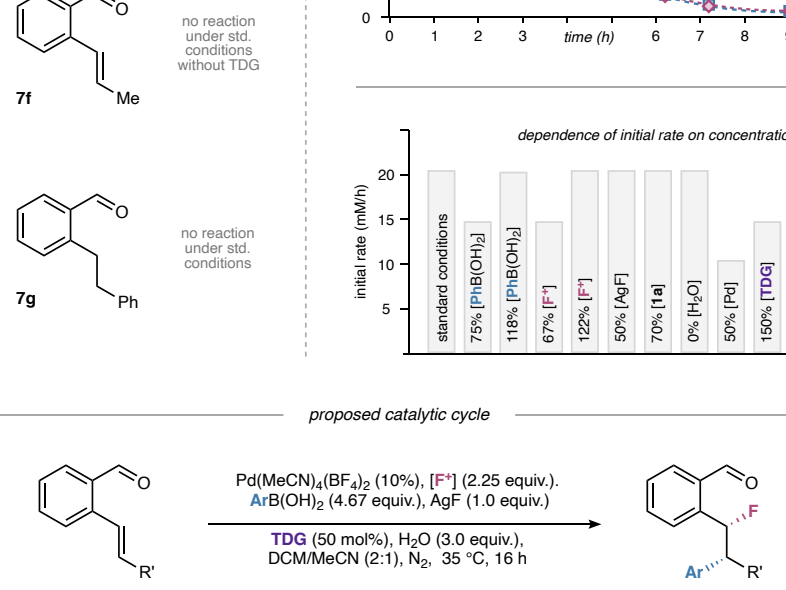

35
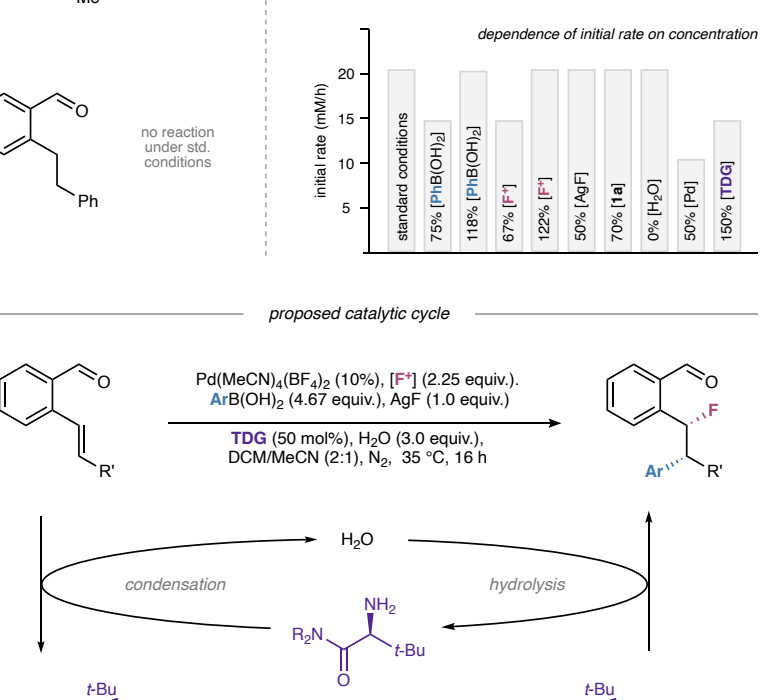

proposed catalytic cycle

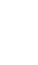
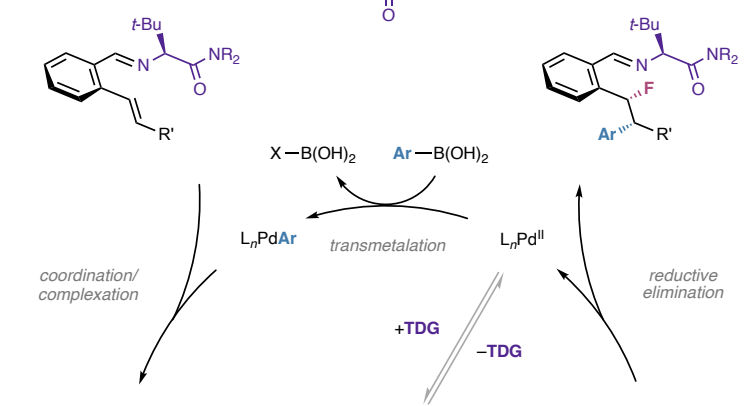

5

60
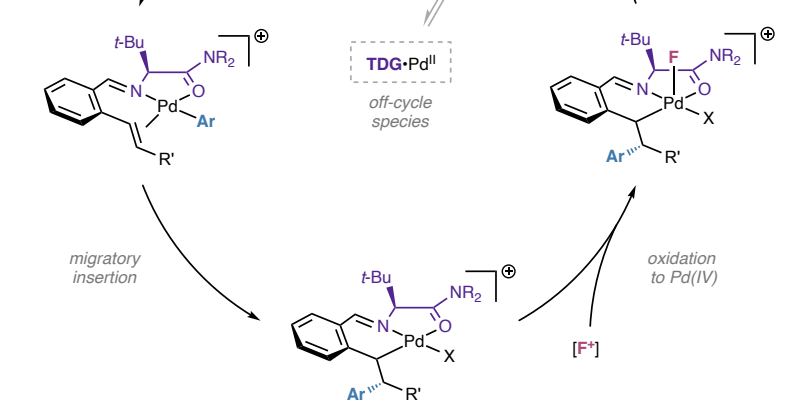

5

Figure 2 | Mechanistic investigation. Summary of control experiments and kinetics investigation (see SI for experimental details and all data) and proposed catalytic cycle. 